\title{
Measurement and modeling of pulsatile flow in microchannel
}

\author{
Mukul Tikekar · Shiv Govind Singh • \\ Amit Agrawal
}

Received: 12 March 2010/ Accepted: 17 May 2010/Published online: 3 June 2010

(C) Springer-Verlag 2010

\begin{abstract}
An experimental study of pulsatile flow in microchannel is reported in this paper. Such a study is important because time-varying flows are frequently encountered in microdevices. The hydraulic diameter of the microchannel is $144 \mu \mathrm{m}$ and deionized water is the working fluid. The pressure drop across the microchannel as a function of time is recorded, from which the average and r.m.s. pressure drops are obtained. The experiments have been performed in the quasi-steady flow regime for a wide range of flow rate, frequency of pulsations, and duty cycle. The results suggest that the pressure with pulsations lies between the minimum and maximum steady state pressure values. The average pressure drop with pulsation is approximately linear with respect to the flow rate. The theoretical expression for pressure has also been derived wherever possible and the experimental data is found to lie below the corresponding theoretical values. The difference with respect to the theoretical value increases with an increase in frequency and a decrease in flow rate, with a maximum difference of $32.7 \%$. This is attributed to the small size of the microchannel. An increase in frequency of square waveform leads to a larger reduction in pressure drop as compared to rectangular waveform, irrespective of the duty cycle. The results can be interpreted with the help of a firstorder model proposed here; the model results are found to compare well against the experimental results. A correlation for friction factor in terms of the other non-dimensional
\end{abstract}

M. Tikekar · A. Agrawal $(\bowtie)$

Department of Mechanical Engineering, Indian Institute of Technology Bombay, Powai, Mumbai 400076, India

e-mail: amit.agrawal@iitb.ac.in

S. G. Singh

Department of Electrical Engineering, Indian Institute

of Technology Hyderabad, Ordnance Factory Estate,

Yeddumailaram 502205, Andhra Pradesh, India governing parameters is also proposed. Experimental study of mass-driven pulsatile flow in microchannel is being conducted for the first time at these scales and the results are of both fundamental and practical importance.

Keywords Microchannel - Pulsatile flow $\cdot$ Microfluidics · Flow modeling $\cdot$ Microscale flow $\cdot$ Pressure drop

\section{List of symbols}

A Area of cross-section of channel $\left(\mathrm{m}^{2}\right)$

$C \quad$ Capacitance (F)

$D_{\mathrm{h}} \quad$ Hydraulic diameter of the channel $(=4 A /$ perimeter $)$ (m)

$f \quad$ Average friction factor $\left(p D_{\mathrm{h}} / \frac{1}{2} \rho v^{2} L\right)$

$f_{\text {rms }} \quad$ Friction factor r.m.s $\left(p_{\text {rms }} D_{\mathrm{h}} / \frac{1}{2} \rho v^{2} L\right)$

$F \quad$ Dimensionless form of frequency $\left(=\frac{D_{\mathrm{h}}}{\left(t_{\mathrm{N}}+t_{\mathrm{F}}\right) \alpha}\right)$

$i \quad$ Current (A)

$L \quad$ Length of the microchannel (m)

$m_{\mathrm{F}} \quad$ Minimum flow rate $(\mathrm{kg} / \mathrm{s})$

$m_{\mathrm{N}} \quad$ Maximum flow rate $(\mathrm{kg} / \mathrm{s})$

$\tilde{p} \quad$ Pressure $(\mathrm{Pa})$

$p \quad$ Time-averaged pressure drop $(\mathrm{Pa})$

$p_{\text {rms }} \quad$ Root mean square value of the pressure drop $(\mathrm{Pa})$

$Q \quad$ Volumetric flow rate $\left(\mathrm{m}^{3} / \mathrm{s}\right)$

$R \quad$ Resistance (ohm)

Re Reynolds number $\left(=\frac{v D_{\mathrm{h}}}{\alpha}\right)$

$R e_{\mathrm{N}}$ On-time Reynolds number $\left(=\frac{v_{\mathrm{N}} D_{\mathrm{h}}}{\alpha}\right)$

$t \quad$ Time (s)

$t_{\mathrm{F}} \quad$ Off-time. Time for which minimum flow $\left(m_{\mathrm{F}}\right)$ occurs (s)

$t_{\mathrm{N}} \quad$ On time. Time for which maximum flow $\left(m_{\mathrm{N}}\right)$ occurs (s)

$T \quad$ Duty cycle or ratio of on-time to off-time $\left(t_{\mathrm{N}} / t_{\mathrm{F}}\right)$

$u \quad$ Streamwise velocity $(\mathrm{m} / \mathrm{s})$ 
$v \quad$ Time-averaged velocity of the flow over the crosssection $(\mathrm{m} / \mathrm{s})$, normalized voltage

V Voltage (V)

$v_{\mathrm{F}} \quad$ Average velocity over the cross-section, during the state of minimum flow $(\mathrm{m} / \mathrm{s})$

$v_{\mathrm{N}}$ Average velocity over the cross-section, during the state of maximum flow $(\mathrm{m} / \mathrm{s})$

$x \quad$ Streamwise coordinate $(\mathrm{m})$

$\alpha \quad$ Kinematic viscosity of the fluid $\left(\mathrm{m}^{2} / \mathrm{s}\right)$

$\beta \quad$ Womersley parameter $\left(=D_{\mathrm{h}} \sqrt{\frac{\pi}{2 \alpha\left(t_{\mathrm{N}}+t_{\mathrm{F}}\right)}}\right)$

$\mu \quad$ Dynamic viscosity of the fluid (Pa.s)

$\omega^{\prime} \quad$ Dimensionless form of frequency $\left(=\frac{D_{\mathrm{h}}}{4\left(t_{\mathrm{N}}+t_{\mathrm{F}}\right) \alpha}\right)$

$\rho \quad$ Fluid density $\left(\mathrm{kg} / \mathrm{m}^{3}\right)$

$\tau_{\mathrm{F}} \quad$ Time constant during the off-phase (s)

$\tau_{\mathrm{N}} \quad$ Time constant during the on-phase (s)

\section{Introduction}

Study of pulsatile flow is particularly relevant at the small scales because of their potential applications in microdevices. For example, numerical simulations by Tang et al. (2006) indicate that a proper combination of electroosmotic flow and pulsatile flow can achieve complete mixing of two microchannel flows. Stroock et al. (2002) and Glasgow and Aubry (2003) observed that inertial effects become important for microfluidic mixing for $R e \gg 1$, which is the case studied here. Further study by Glasgow et al. (2004a, b) showed that the nature of flow affects the degree of mixing and that intermediate flows have a higher degree of mixing as compared to quasi-steady flows. Apart from mixing, Steinke and Kandlikar (2004) proposed that pulsations in microchannel flows may enhance single phase heat transfer, thus aiding in electronics cooling. A similar observation has been reported by Havemann and Rao (1954) in the context of turbulent flows. Pulsatile flows have also been used by $\mathrm{Gu}$ et al. (2004) in a computer-controlled microfluidic cellular assay powered by a refreshable Braille display. Unger et al. (2000) introduced the idea of multilayer soft lithography and fabricated a peristaltic micropump operating on pulsatile flows. Pulsating magneto-hydrodynamic micropumps have been theoretically studied and extensively analyzed by Duwairi and Abdullah $(2007,2008)$ and Abdullah and Duwairi (2008a, b). The importance of pulsatile flow cannot be overemphasized because these are widespread in fluid systems at the microscales.

Although the first study on pulsatile flow was undertaken by Sexl as early as in 1930, there is still lot to be desired in terms of a comprehensive understanding of this class of flows. A review of pulsatile flow studies conducted in the previous century was undertaken by Çarpinlioglu and Gündogdu (2001) which indicates that there is a paucity of detailed experimental data even for basic configurations.
Moreover, a fundamental study investigating the effect of pulsations on such basic quantities such as overall pressure drop is missing at the microscales. This study provides pressure drop data for pulsatile flow of water in rectangular microchannel. This is among the first such experimental study at the microscales, although some theoretical results with gas flow in microchannel (involving slip at the wall) are available (Chakraborty and Bhalla 2007; Wu et al. 2008). Some very recent theoretical studies (Brereton 2009; Haddad et al. 2010) have revisited the problem. These studies, similar to other theoretical reports, predict a symmetric variation in pressure, which is not brought out by measurements in the present work, suggesting differences in flow behavior at microscales with respect to conventional understanding.

The governing equation for pulsatile flow is (see example, Yakhot et al. 1999):

$\frac{\partial u}{\partial t}=-\frac{1}{\rho} \frac{\partial \tilde{p}}{\partial x}+\alpha \nabla^{2} u$.

Based on the above equation, pulsatile flow systems are divided into three regimes, namely, quasi-steady, intermediate, and inertia-dominant, depending on the nondimensional pulsation frequency value. In the quasi-steady regime, the pressure term is balanced by viscosity and the unsteady term in Eq. 1 is negligibly small; this happens at low frequencies $\left(\sqrt{\omega^{\prime}} \leq 1.32\right.$, Ohmi et al. 1981). In the inertia-dominant regime, the pressure term is primarily balanced by the unsteady term while viscous effects are confined to near-wall regions; this happens at high frequencies $\left(\sqrt{\omega^{\prime}} \geq 28\right.$, Ohmi et al. 1981). A phase difference of $90^{\circ}$ between wall shear stress and pressure, and $45^{\circ}$ between average velocity and pressure, exists in the latter domain; there is, however, no phase difference in the quasi-steady regime (Ohmi et al. 1981; Yakhot et al. 1999). In certain cases the mass flow rate is prescribed and the variation in pressure with time needs to be determined; whereas a known pressure gradient is applied in other cases. As pointed out by Das and Arakeri (2000); Ünsal et al. (2005), among others, there are several differences between the two situations. The former is applicable, for example, to opening/closing of a valve, flow induced by motion of a piston, among other cases, and is the lesser studied of the two situations. In such a case, Eq. 1 needs to be solved along with the constraint that

$\int_{A} u \mathrm{~d} A=Q(t)$

where $Q(t)$ is volume flow rate prescribed as some function of time (Das and Arakeri 2000). Some attempts at modeling the flow as a low-order system is also available. For example, Morris and Forster (2004) modeled the flow as a resistor and inductor connected in series (see also Nichols 
and O'Rourke 1990); the difficulty in obtaining the value of inductance was noted in their study. As per the above classification, the present study lies primarily in the quasisteady flow domain. The flow is always laminar.

A paucity of studies on pulsatile flow is brought about by the above review. In particular, experimental data on pulsating flow in microchannels is missing. The specific objectives of the present study are:

1. To provide pressure drop measurements on pulsating flow in microchannel. These are among the first such measurements in the literature.

2. To develop a correlation for friction factor in terms of the other non-dimensional parameters. The correlation can be used in design of microdevices involving pulsatile flow.

3. To develop a low-order system model to help interpret the data. The model can be used with care to predict the flow behavior beyond the parameter range covered in the experiments.

4. To bring out differences in the results as compared to those reported in the literature under somewhat similar conditions. This should help enhance the physical understanding of the problem.

In the present work, square or rectangular variation in mass is imposed on flow through a microchannel, and pressure as a function of time is measured, from which the mean and r.m.s. pressure drops are obtained. The measurements are performed for a wide range of input parameters such as average flow rate, frequency of pulsations, and duty cycle (square or rectangular input pulse). In rectangular pulse, the effect of holding one time duration constant while changing the other is also systematically studied. The results lead to a correlation for friction factor in terms of the other nondimensional parameters. Theoretical expressions are derived wherever possible and a model is presented to help analyze the results further. A comparison of the theoretical and model results with the experimental data provides useful insights about the flow physics.

\section{Experimental setup and validation}

The system under study consists of a single rectangular microchannel $(260 \mu \mathrm{m} \times 100 \mu \mathrm{m}$ in cross-section and $20 \mathrm{~mm}$ in length) with reservoirs at its two ends. The output reservoir is open to the atmosphere, and therefore, the pressure drop across the microchannel is obtained by recording the gauge pressure at the input of the microchannel. The pressure gauge (Keller, Leo 1) has a range of -1 to 3 bar with a least count of 1 mbar, and a response time of $1 \mathrm{~s}$. As evident from the results presented in later sections, the response time of the system is of the order of minutes. The sampling rate of $1 \mathrm{~s}$ is therefore adequate to study the pressure drop characteristics of the system with pulsations. The pressure gauge is connected to a PC through a data logger. Deionised water is supplied from a container with the help of a micropump (Masterflex Easyflow II EW-77200-50 with a range of 0.1-6 ml/min, a resolution of $0.01 \mathrm{ml} / \mathrm{min}$ and a maximum pressure head of 1.7 bar). The microchannel is fabricated in-house on a silicon wafer; the fabrication details can be found elsewhere (Singh et al. 2008, 2009).

The incoming flow is split into two lines (Fig. 1), one of which passes through the microchannel under consideration and the other goes through solenoid valve which serves as the generator of the pulsating flow. The solenoid valve is a computer controlled on/off device. It can be programmed to remain on (open) for a specified period of
Fig. 1 Schematic diagram of the experimental setup

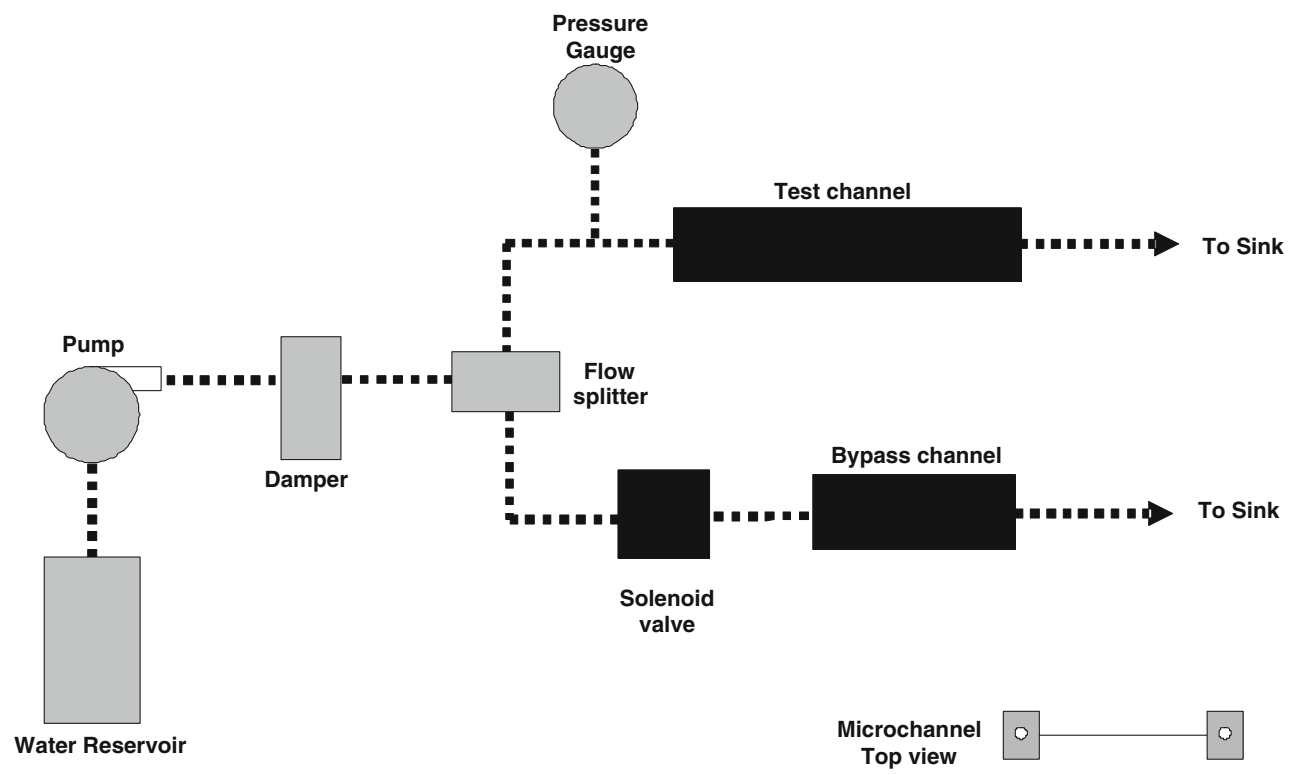


time (maximum duration of $100 \mathrm{~h}$ and a minimum of $1 \mathrm{~s}$ with a resolution of $1 \mathrm{~s}$ ) and then stay off (closed) for another fixed period of time. When the valve is closed, all the flow pumped from the reservoir passes through the test channel. This state is referred to as the 'on' state of flow and is designated by the subscript ' $N$ '. When the valve is open, part of the flow passes through the test channel. The other part passes through the solenoid valve, through a bypass channel to another atmospheric sink. This state is called the 'off' state of flow and is designated by the subscript ' $F$ '. The opening/closing of the value results in rectangular pulses (see Fig. 2a). Since the test microchannel offers much higher resistance to the flow as compared to the valve, an additional resistance, comparable to the test microchannel resistance, is connected in series with the valve. This bypass microchannel ensures that the ratio of
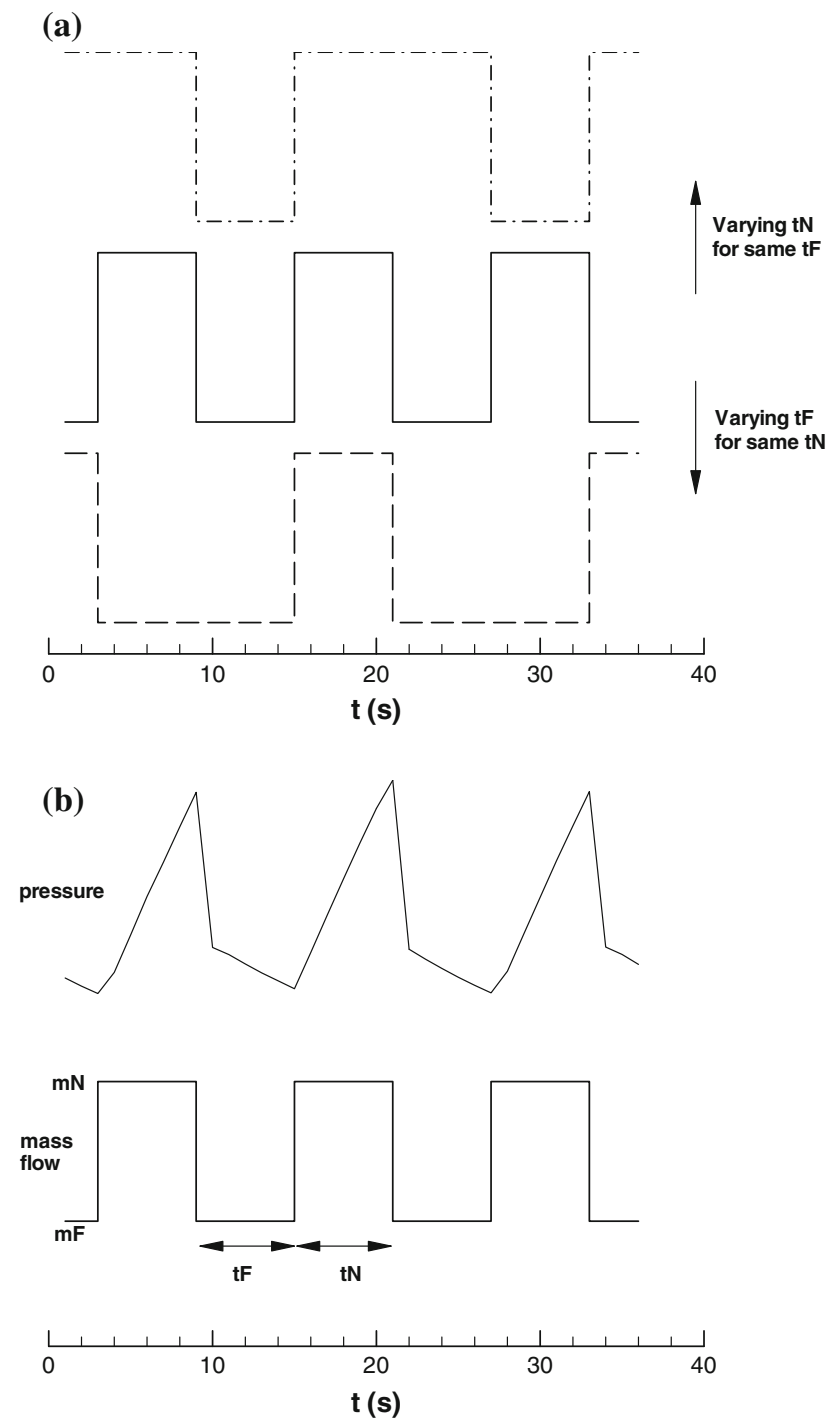

Fig. 2 a Definition of various parameters and $\mathbf{b}$ their effect on the flow flow passing through the test channel to that passing through the bypass microchannel remains constant and finite. Note that the same bypass microchannel has been employed for all experiments performed herein. The response time of the solenoid valve is about three orders of magnitude smaller than the period of imposed pulsations. The length of all connecting tubes is kept at the minimum possible.

The system is studied in one of the following ways (Fig. 2):

1. Varying the mass flow rate through the system, keeping the on- and off-times constant: This is done by changing the setting of the pump while the solenoid valve executes the same cycle.

2. Varying the period of pulsation with square waveform, keeping the mass flow rate same: The solenoid valve can be programmed to remain open and close for set periods of time. Suitable combinations of the on- and off-times are chosen such that their ratio $\left(t_{\mathrm{N}} / t_{\mathrm{F}}\right)$ remains the same.

3. Changing the duty cycle by varying the on-time while keeping the off-time same: As mentioned above, the solenoid value can be set to remain open and close for any pre-specified duration. The mass flow rate is kept constant by keeping a fixed setting of the micropump (see Fig. 2a).

4. Changing the duty cycle by varying the off-time while keeping the on-time same: This setting, too, is controlled as available in the solenoid valve and is similar to point 3 above.

As presented later, points 3 and 4 above yield different results. Note that the amplitude of pulsations $\left(m_{\mathrm{F}} / m_{\mathrm{N}}\right)$ can be varied by changing the resistance of the by-pass line. However, due to practical considerations $m_{\mathrm{F}} / m_{\mathrm{N}}$ has been fixed at 0.578 and such experiments were not undertaken.

The mass flow rate supplied by the pump is varied between 0.1 and $4 \mathrm{ml} / \mathrm{min}$, time period of pulsation is varied between 8 and $240 \mathrm{~s}$, and the waveforms tested are square and rectangular. With rectangular waveform, either the on- or off-time is held constant (at $24 \mathrm{~s}$ ), while the other is varied between 4 and $144 \mathrm{~s}$. For a microchannel of hydraulic diameter of $144 \mu \mathrm{m}$, this leads to $R e=10-620$, $F=0-0.00365$, and $T=0.17-6$. (The non-dimensional numbers, $R e, F$, and $T$ are defined later through Eqs. 6-8.) Note that the residence time of flow in the microchannel is of the order of $10 \mathrm{~ms}$ which is three-four orders of magnitude smaller than the time period of pulsation. Such vast difference in the two time-scales is expected at microscales and suggests that pulsatile flow in microchannel will mostly lie in the quasi-steady regime. It is difficult to achieve non-quasi-steady flow in microchannels, because of the strong viscous effects brought about by the small 
dimension of the microchannel. Very high frequency pulsations (of the order of $\mathrm{kHz}$ ) need to be superimposed to overcome the effect of small dimension. Such high frequencies are difficult to work with because of a reduction in time scale for both actuation and measurement. The maximum uncertainty in Reynolds number and friction factor are estimated at 5.4 and $10.6 \%$, respectively.

The developing length of the flow as a percentage of the microchannel length is an important parameter in such studies. According to $\mathrm{He}$ and $\mathrm{Ku}$ (1994) the flow entrance length is approximately the same as steady entrance length for low Womersley number flows, which is the case in the present work. Lee et al. (2002) showed that the entrance length for steady flows can be calculated by the equation $0.033 \times D_{\mathrm{h}} \times R e$ for Reynolds number ranging from 200 to 2100 . Using this expression, the maximum entrance length for the present set of experiments is about $2.9 \mathrm{~mm}$. As per the correlation provided by Lee et al. (2008), the maximum entrance length comes out to be $2.4 \mathrm{~mm}$. Therefore, for most of the cases the development length is less than $15 \%$ of the total length $(=20 \mathrm{~mm})$, which is within the acceptable limits. Indeed no effect of entrance length on pressure drop is apparent from the results as well (for example in Fig. 4 presented later).

Before studying the transient characteristic of the system, the steady state pressure drop values were checked. For this, pressure drop as a function of mass flow rate is recorded. These are converted to non-dimensional formfriction factor $(f)$ and Reynolds number $(R e)$. As shown in Singh et al. $(2008,2009)$ the experimentally obtained values of $f \cdot R e$ compare well against the theoretical calculations of Morini (2004). This helps to validate the experimental setup and the measurement techniques.

\section{Measurement of pressure drop}

The result for pressure drop by varying single parameter at a time while holding the other parameters constant is presented in this section.

\subsection{Effect of flow rate}

A typical pressure-time series for different flow rate values, with total pulse duration of $12 \mathrm{~s}$ and $t_{\mathrm{N}} / t_{\mathrm{F}}=1$ (square pulse) is shown in Fig. 3a. The pressure increases linearly over the duration $(6 \mathrm{~s})$ of maximum flow rate (see also Fig. 2b). With a reduction in flow rate, the pressure drop first decreases rapidly than exhibits a rather slow reduction. This observation applies to all flow rates; an explanation for this behavior is offered later in Sect. 5.1. Comparing Fig. $3 \mathrm{a}$ with $\mathrm{b}$, where the pulse duration is increased to $24 \mathrm{~s}$, while the other parameters are held
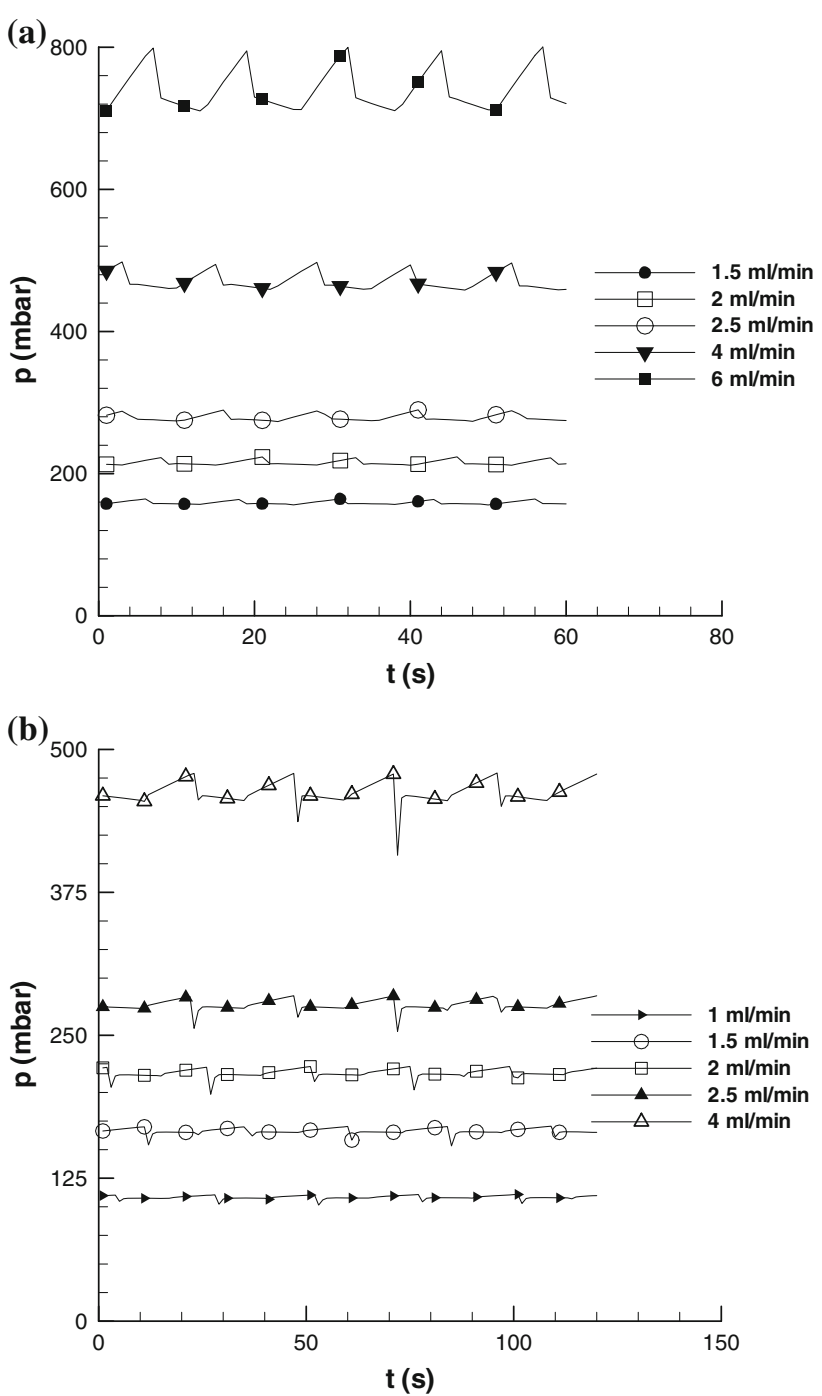

Fig. 3 Pressure drop as a function of time for different mass flow rates, keeping the duration of square pulse as a $12 \mathrm{~s}$ and $\mathbf{b} 24 \mathrm{~s}$

constant, reveals that the basic nature of the waveform has not changed. The pressure, however, drops rapidly at the beginning of off-time and may exhibit an undershoot in the latter case.

The above results suggest that the time required for the pressure to ramp up is not sufficient in this case. Other experiments where the durations of both the on- and offtimes are increased are presented later; these show that the time required for the pressure to build up is about $5 \mathrm{~min}$ for a flow rate of $2 \mathrm{ml} / \mathrm{min}$.

Figure 4 shows that the average pressure drop increases linearly with flow rate, i.e., the pulsatile flow behaves similar to a steady flow in this respect. The slope is, however, slightly smaller with pulsations, as evident upon comparing against the theoretical line. This suggests reduction in pumping power when the flow is pulsating; 

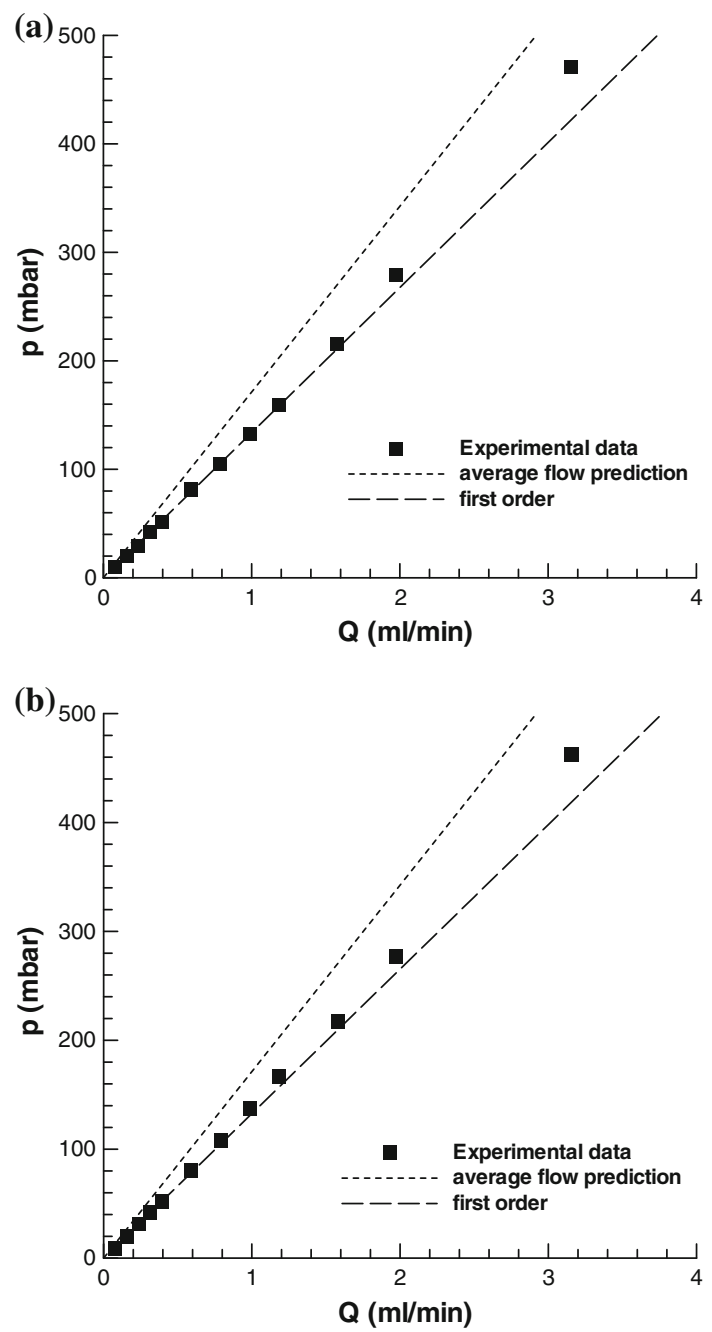

Fig. 4 Variation in mean pressure as a function of flow rate, keeping the duration of square pulse as a $12 \mathrm{~s}$ and b $24 \mathrm{~s}$

this reduction, however, comes at the cost of additional power required to create the pulsations. The estimated saving in pumping power is between 0 and $0.2 \mathrm{~mW}$ while the additional power required to operate the solenoid valve is about $4 \mathrm{~W}$. These values suggest that the coefficient of performance of the system is poor. The performance can be improved by employing a microvalue, with a substantially smaller input power. However, optimization of energy efficiency of the system is beyond the scope of the current work. The result from first-order model is also included in the figure; this is discussed in detail later in Sect. 5.2.

The pressure r.m.s increases almost quadratically with the mass flow rate (Fig. 5). The pressure fluctuations normalized by the average pressure $\left(p_{\mathrm{rms}} / p\right)$ is $1.28 \%$ at $Q=1 \mathrm{ml} / \mathrm{min}$ and $2.5 \%$ at $Q=4 \mathrm{ml} / \mathrm{min}$. The relatively small value of normalized fluctuation is noteworthy; this is attributed to the underlying flow being laminar and quasisteady.
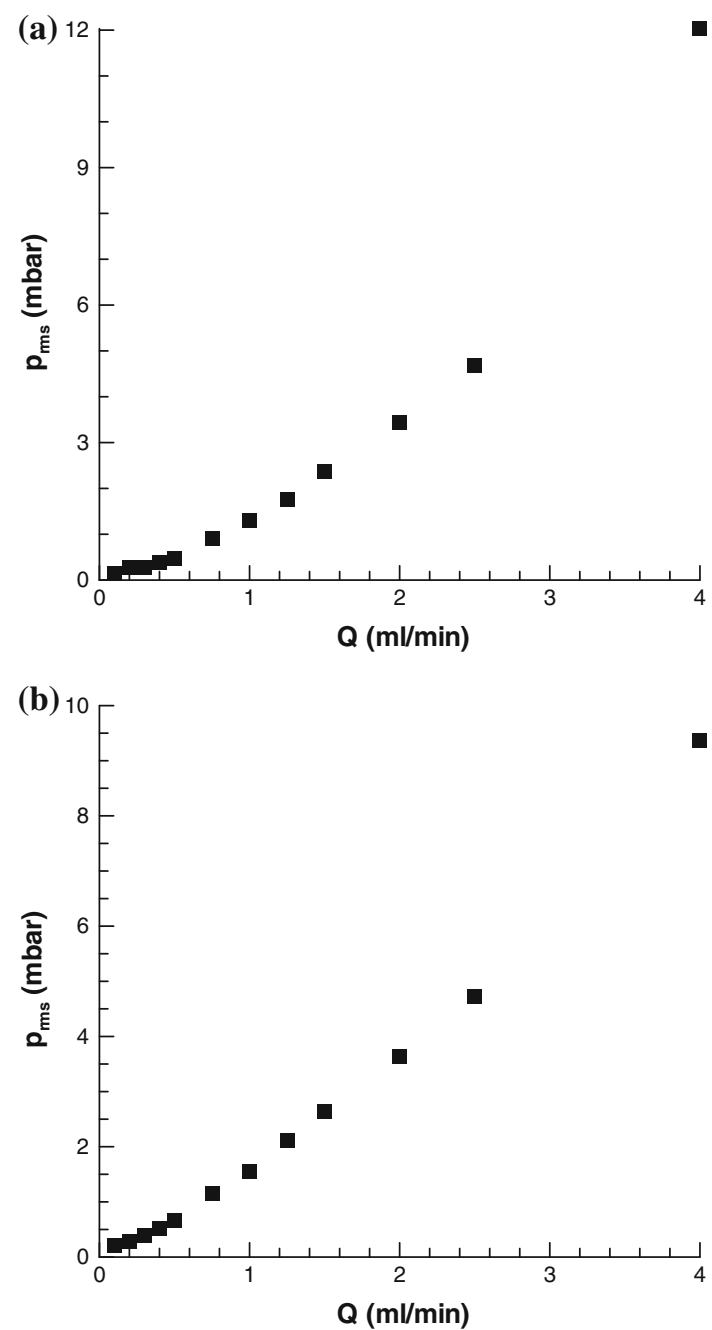

Fig. 5 Variation in pressure r.m.s. as a function of flow rate, keeping the duration of square pulse as a $12 \mathrm{~s}$ and $\mathbf{b} 24 \mathrm{~s}$

\subsection{Effect of time period}

The effect of varying the time period of pulsation for a square waveform $\left(t_{\mathrm{N}} / t_{\mathrm{F}}=1\right)$ with a constant on flow rate of $2 \mathrm{ml} / \mathrm{min}$, is studied in this section. The pressure-time series shows that the resulting pressure has the same frequency as that imposed on the system. For the cases considered herein, the minimum pressure drop is approximately the same (although this may not be true in general); however, the maximum pressure drop increases with an increase in the pulse duration (Fig. 6). This is because with an increase in the period of pulsation there is more time for pressure to attain the pressure levels corresponding to the minimum and maximum mass flow rates. Because of this, both the average pressure drop and pressure r.m.s. increases monotonically with the period of pulsation; see Figs. 7 and 8. The exponential rise and fall in pressure evident from Fig. 6 suggests that the system can be modeled as a first-order system (this 


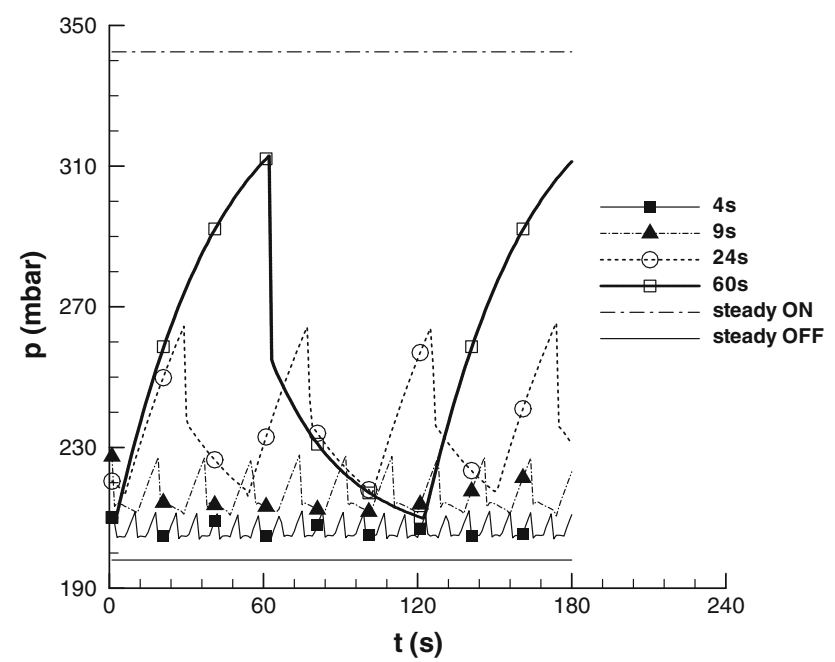

Fig. 6 Pressure versus time for different on-times, for square pulsations with a flow rate of $2 \mathrm{ml} / \mathrm{min}$. The two horizontal lines represent the maximum (i.e., steady state on) and minimum (i.e., steady state off) attainable pressures

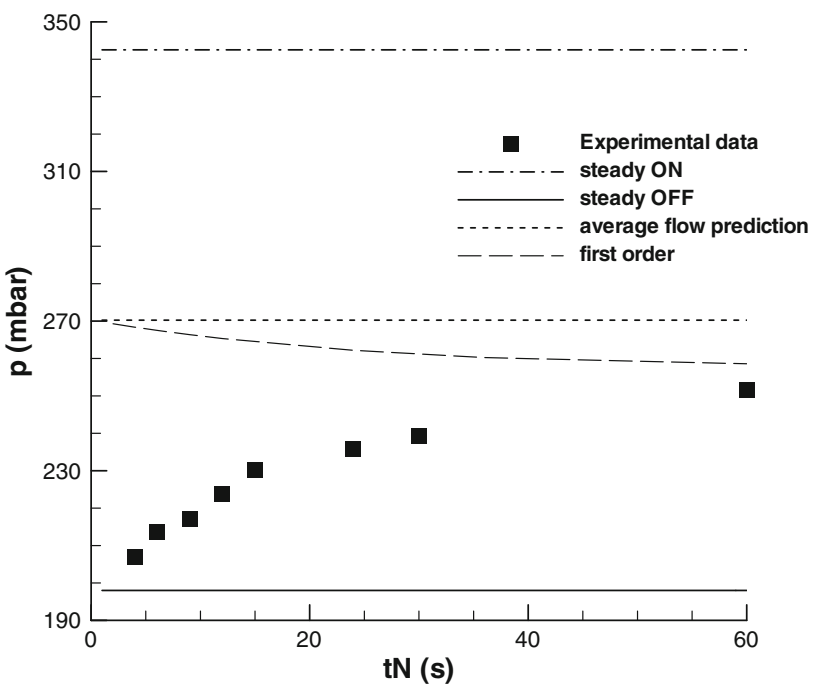

Fig. 7 Variation of average pressure with on-time, for square pulsations with a flow rate of $2 \mathrm{ml} / \mathrm{min}$. The dotted line represents the time-weighted average pressure of the on and off steady state pressures, while the short dash-dot and solid lines indicate the maximum and minimum attainable pressure drop values, respectively. The long dashed line is from first-order model

point is discussed further in Sect. 5). That is, over- and/or under damping behavior, which is typical of second-order system, is not observed here.

As compared to the theoretical value (short dashed line in Fig. 7), the average pressure drop decreases by about $24 \%$ for small time periods of oscillation. Note that this variation in pressure drop with time period occurs even when the average mass flow rate is the same for all the cases. The pr essure drop tends to saturate for larger time period of pulsations, and attains a value $7 \%$

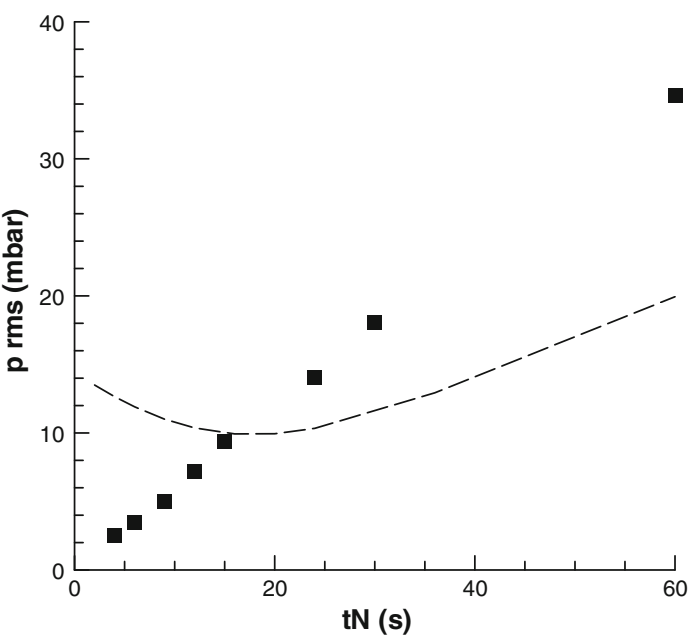

Fig. 8 Variation of pressure r.m.s with on-time, for square pulsations with a flow rate of $2 \mathrm{ml} / \mathrm{min}$. The first-order model result is also included as dashed line

less than the pressure drop value corresponding to the average flow rate. The increase in pressure r.m.s. is found to be linear passing through the origin (Fig. 8) unlike average pressure which exhibits a square-root like variation. The pressure r.m.s. is expected to asymptote to a constant value upon further increasing the time period of oscillation; this is, however, not very evident from the figure.

\subsection{Effect of duty cycle for the same off-time}

A square waveform has been applied in the above sections. The effect of changing the waveform from square to rectangular is now considered. The variation in duty cycle of the solenoid valve (i.e., changing either the on- or off-time, keeping the other duration constant) is studied in the following two sections. It will be seen that changing the duty cycle in these two different ways lead to different behavior. The present work covers a sufficiently large range, and alludes to the rise and fall of pressure during transients. This provides an idea of the response time and other characteristics of the system.

The pressure-time series, where the off-time is maintained constant at $24 \mathrm{~s}$ (arbitrarily chosen) while the on-time is varied between 4 and $144 \mathrm{~s}$, for a flow rate of $2 \mathrm{ml} / \mathrm{min}$, is shown in Fig. 9. The corresponding steady state pressure drop values are also shown in the figure. The pressure lies between the two steady state values for all the cases, with an increase in both the minimum and maximum values with increasing on-time. Further, the pressure drop is rapid at the beginning of the off-time for $t_{\mathrm{N}} \leq t_{\mathrm{F}}$, and gradual otherwise. The pressure is found to increase continuously (with a 


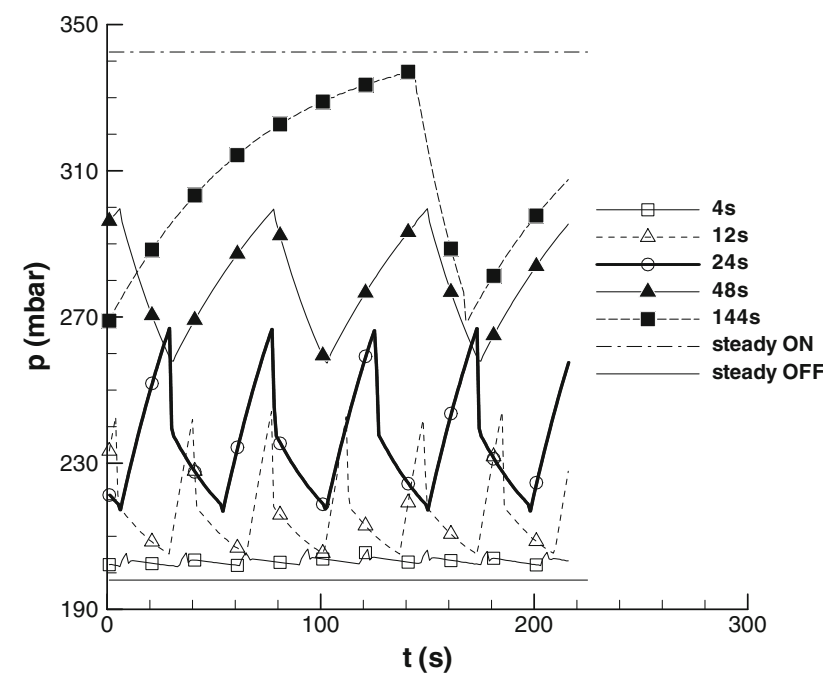

Fig. 9 Pressure versus time for different on-times at constant offtime of $24 \mathrm{~s}$, and a maximum flow rate of $2 \mathrm{ml} / \mathrm{min}$. The two horizontal lines represent the maximum (i.e., steady state on) and minimum (i.e., steady state off) pressures

decreasing rate) during the on-period for $t_{\mathrm{N}}=144 \mathrm{~s}$. This case also reveals that although the pressure rises nonlinearly, it would appear linear for small $t_{\mathrm{N}}$ values.

Figure 10 shows that the average pressure increases monotonically with an increase in the on-time. This is related to an increase in the average flow rate with an increase in the on-time. The predicted pressure drop corresponding to the average flow rate is given by

$p=\left(\frac{31.2 \mu L}{\rho A D_{\mathrm{h}}^{2}}\right) \cdot m_{\mathrm{avg}}$

where $m_{\mathrm{avg}}$ is the average mass flow rate through the microchannel, given by

$m_{\mathrm{avg}}=\frac{m_{\mathrm{N}} t_{\mathrm{N}}+m_{\mathrm{F}} t_{\mathrm{F}}}{t_{\mathrm{N}}+t_{\mathrm{F}}}$

The theoretical curve is also plotted in the figure (as short dashed line). Interestingly, all experimental points are below the theoretical curve with a maximum difference of $12.6 \%$ for $t_{\mathrm{N}}=t_{\mathrm{F}}=24 \mathrm{~s}$. This suggests that the actual pressure drop with pulsations is lesser than the expected (theoretical) value. The experimental pressure heads to a saturation value as also suggested by the above equations. This is related to the fact that, as the on-time increases, the flow approaches its maximum value.

Figure 11 shows that the pressure r.m.s. also increases (almost monotonically) with an increase in the on-time. This is related to the fact that both the minimum and maximum pressure values increase monotonically with an increase in the on-time, but the maximum value increases more than the minimum value.
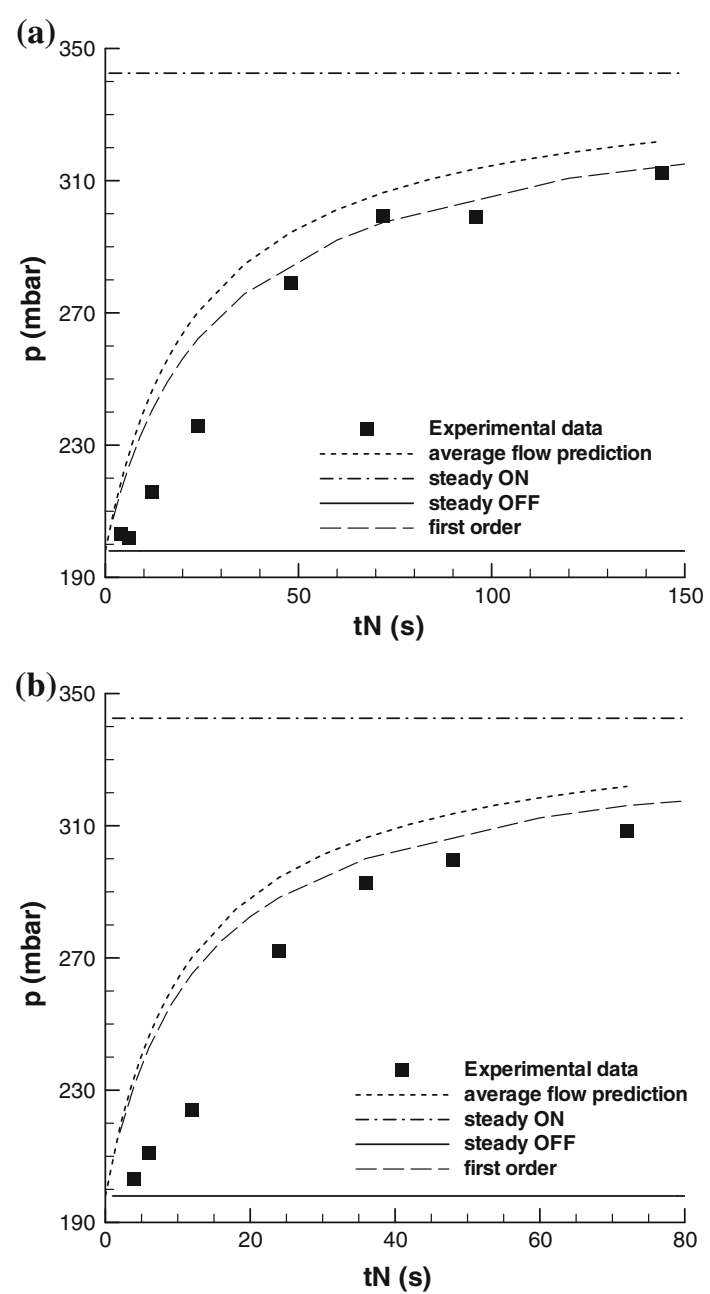

Fig. 10 Variation in mean pressure as a function of on-time, keeping the mass flow rate (both minimum and maximum) and off-time constant at $\mathbf{a} 24 \mathrm{~s}$ and $\mathbf{b} 12 \mathrm{~s}$. The dash-dot and solid lines correspond to the maximum and minimum steady state values, while the dotted line represents the corresponding theoretical values. The dashed line is from first-order model

\subsection{Effect of duty cycle for the same on-time}

The pressure-time series where the on-time is maintained constant at $24 \mathrm{~s}$ while the off-time is varied between 4 and $144 \mathrm{~s}$, is shown in Fig. 12. The corresponding steady state pressure drop values are also shown in the figure. The pressure lies between the two steady state values for all the cases and at all times. The pressure is found to decrease continuously during the off-period for $t_{\mathrm{F}}=144 \mathrm{~s}$ case. As noted earlier, the pressure drop is rapid at the beginning of the offtime for $t_{\mathrm{N}} \leq t_{\mathrm{F}}$, and gradual otherwise. However, unlike in the previous case, both the minimum and maximum values change non-monotonically with a change in the on-time.

Figure 13 shows that the average pressure decreases monotonically with an increase in the off-time. This is related to a decrease in the average flow rate with an increase 

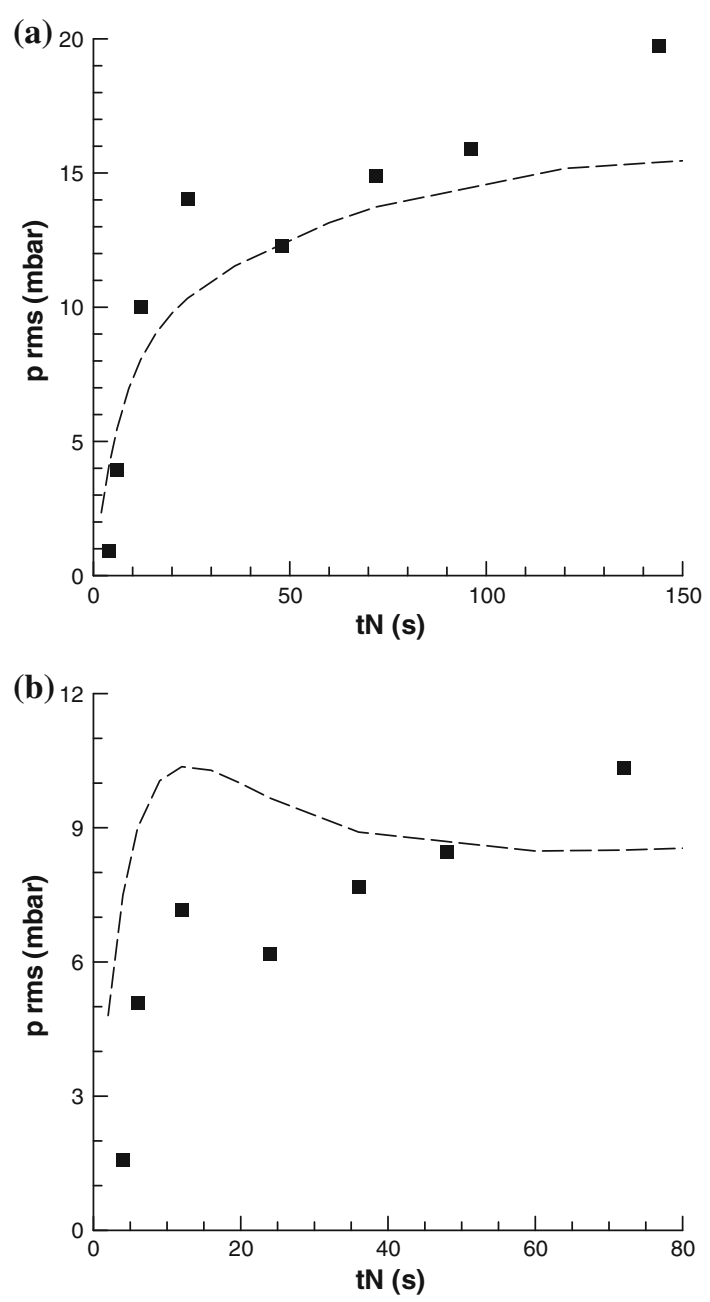

Fig. 11 Variation in pressure r.m.s. as a function of on-time, keeping mass flow rate (both minimum and maximum) and the off-time constant at a $24 \mathrm{~s}$ and $\mathbf{b} 12 \mathrm{~s}$. The first-order model result is also included as dashed line

in the off-time. The theoretical pressure drop in terms of the average flow rate can again be calculated using Eqs. 3 and 4 . As before, the experimentally measured pressure drop is less than the theoretical value with the maximum difference of $16 \%$ occurring for $t_{\mathrm{N}}=t_{\mathrm{F}}=12 \mathrm{~s}$. The pressure tends to saturate as the flow approaches the minimum flow steady state value in accord with our earlier discussion.

Figure 14 shows a non-monotonic variation in pressure r.m.s with an increase in the off-time. This non-monotonic variation is clearly related to the minimum and maximum pressure values being non-monotonic with off-time.

\section{Non-dimensional results for pressure drop}

The parameters described above may be studied in their non-dimensional forms. There are four independent nondimensional parameters under question, namely, friction

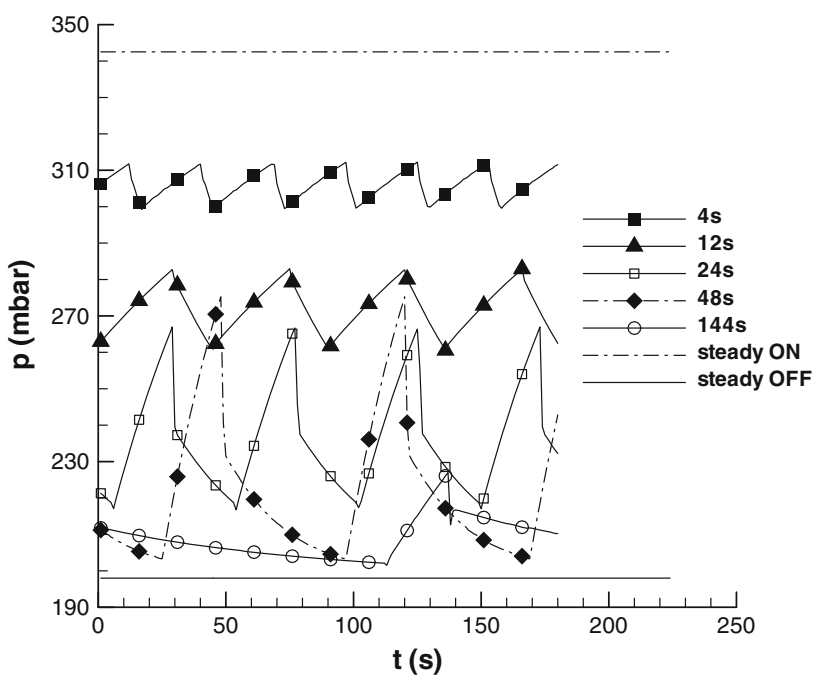

Fig. 12 Pressure versus time for different off-times at constant ontime of $24 \mathrm{~s}$, for a maximum flow rate of $2 \mathrm{ml} / \mathrm{min}$. The two horizontal lines represent the maximum (i.e., steady state on) and minimum (i.e., steady state off) attainable pressures

factor, Reynolds number, non-dimensional frequency, and duty cycle. These parameters are given by the following equations:

$f=\frac{p}{1 / 2 \rho v^{2}} \frac{D_{\mathrm{h}}}{L}$

$R e=\frac{v D_{\mathrm{h}}}{\alpha}$

$F=\frac{D_{\mathrm{h}}^{2}}{\alpha\left(t_{\mathrm{N}}+t_{\mathrm{F}}\right)}$

$T=\frac{t_{\mathrm{N}}}{t_{\mathrm{F}}}$

The effect of the other three non-dimensional parameters on the average and r.m.s. friction factor is presented in this section. Note that besides $F$, Womersley parameter $(\beta)$ and $\omega^{\prime}$ are also used to non-dimensionalize the frequency; these are, respectively, related to $F$ as $F=(2 / \pi) \beta^{2}$ and $F=4 \omega^{\prime}$.

\subsection{Effect of Reynolds number}

Figure 15 shows the effect of Reynolds number on friction factor. Both the average and r.m.s. friction factor decrease monotonically as anticipated from the pressure drop versus flow rate variation. The average friction factor decreases slightly with an increase in the non-dimensional frequency. The average friction factor times the Reynolds number is also plotted for a ready comparison against the corresponding steady state value (Fig. 16). It shows that the $f \cdot R e$ value for pulsatile flow is less than and asymptotes to the steady value, with an increase in Reynolds number. The maximum reduction in $f \cdot R e$ with pulsations is about $32.7 \%$ with 

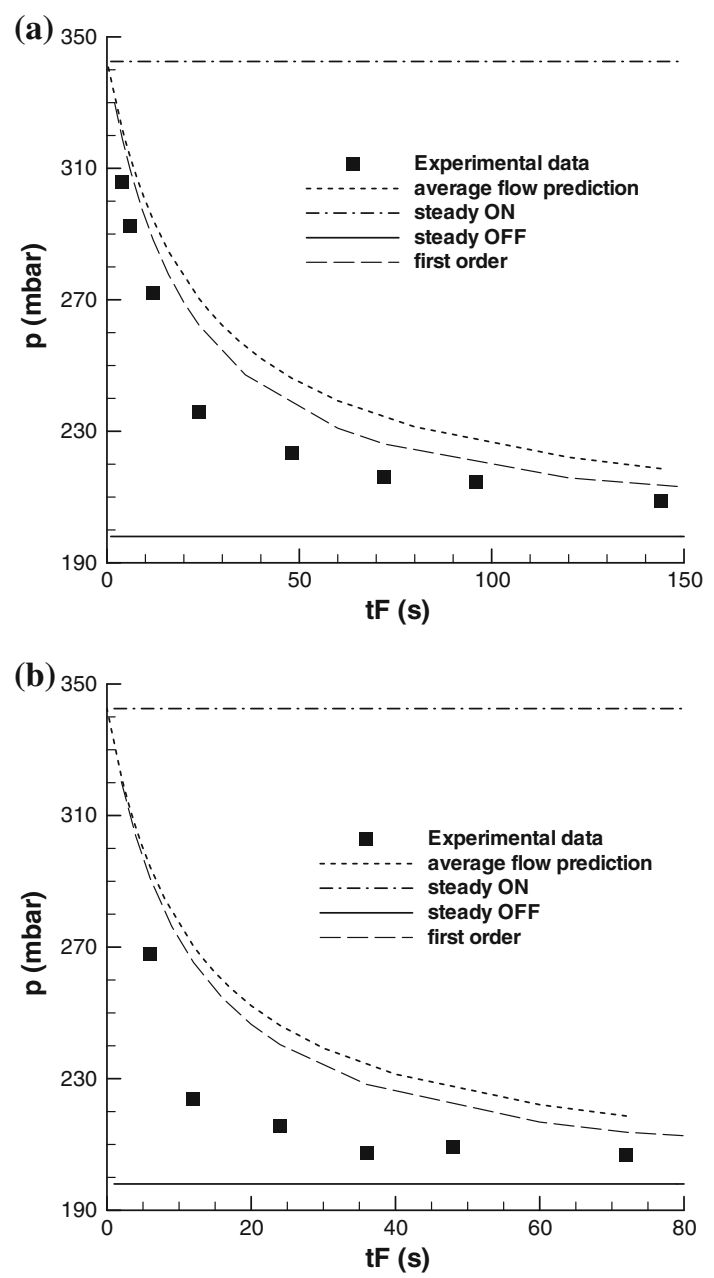

Fig. 13 Variation in mean pressure as a function of off-time, keeping mass flow rate (both minimum and maximum) and the on-time constant at a $24 \mathrm{~s}$ and b $12 \mathrm{~s}$. The dash-dot and solid lines correspond to the maximum and minimum steady state values, while the dotted line represents the corresponding theoretical values. The dashed line is from first-order model

$F=0.000609$ and $R e=10$. A model to explain the frictional behavior of steady liquid flow in microchannels has been recently advanced by Chakraborty et al. (2007).

\subsection{Effect of non-dimensional frequency}

Figure 17a shows that the average friction factor decreases monotonically with an increase in the non-dimensional frequency. Note that the largest drop in friction factor of $24 \%$ is obtained for the square pulse $(T=1)$ and negligible (4-7\%) difference is observed with rectangular pulses with a larger on-time $(T=2,4)$. For $T=0.5$, a drop of $13 \%$ is obtained and an $11 \%$ decrease is found with $T=0.25$. In other words, a square waveform is more sensitive to a change in frequency as compared to rectangular pulse, and the friction factor is almost invariant of frequency if the ontime is greater than the off-time. In the limit of either on- or
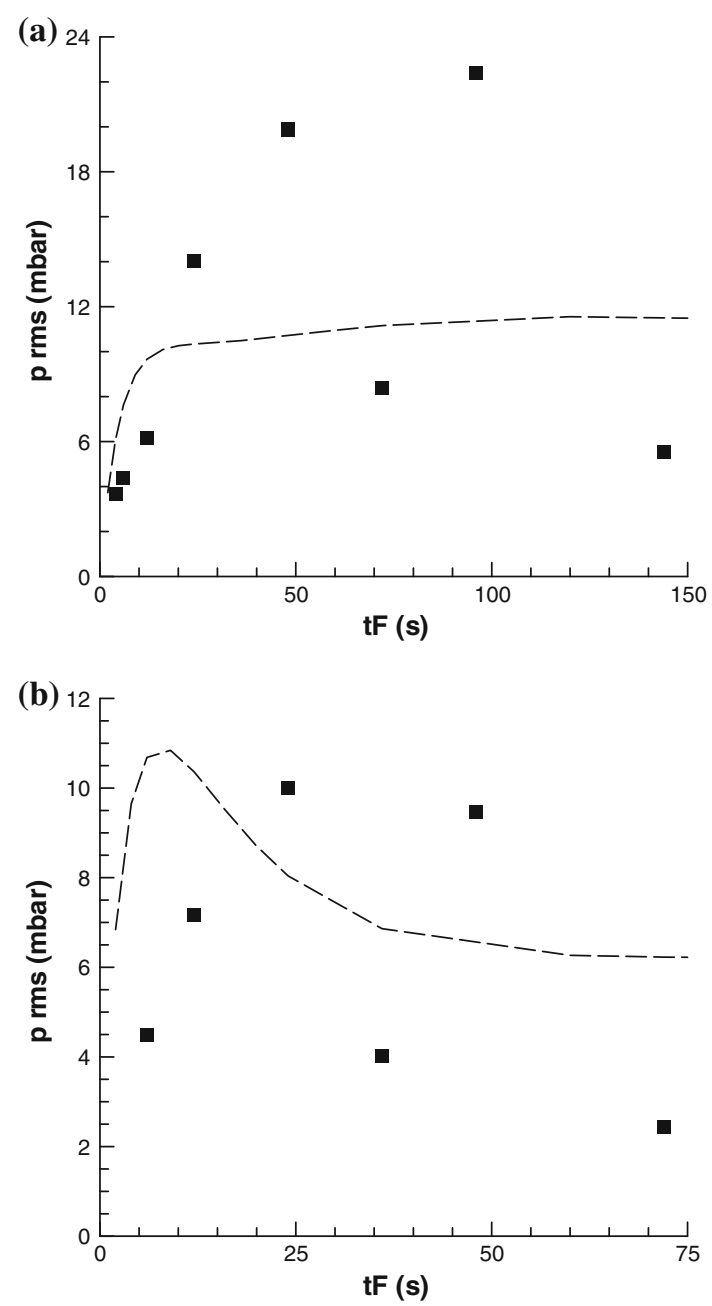

Fig. 14 Variation in pressure r.m.s. as a function of off-time, keeping mass flow rate (both minimum and maximum) and the on-time constant at a $24 \mathrm{~s}$ and b $12 \mathrm{~s}$. The first-order model result is also included as dashed line

off-time going to zero or infinity, the results should become invariant of frequency, and results for the rectangular pulses can be viewed in light of this observation.

Figure 17b shows that the friction factor r.m.s also decreases monotonically with the non-dimensional frequency. It is further noticed that for $T>1$ the friction factor r.m.s is relatively lesser dependent on frequency, in line with that observed for the average friction factor. A simultaneous reduction in both average and r.m.s. friction factor with an increase in frequency is desirable from a practical standpoint.

\subsection{Effect of duty cycle}

The variations of average friction factor and friction factor r.m.s with the ratio of on- to off-time are shown in Figs. 18a and $\mathrm{b}$, respectively. The average friction factor decreases monotonically with increasing on-off times ratio. This is related to an 

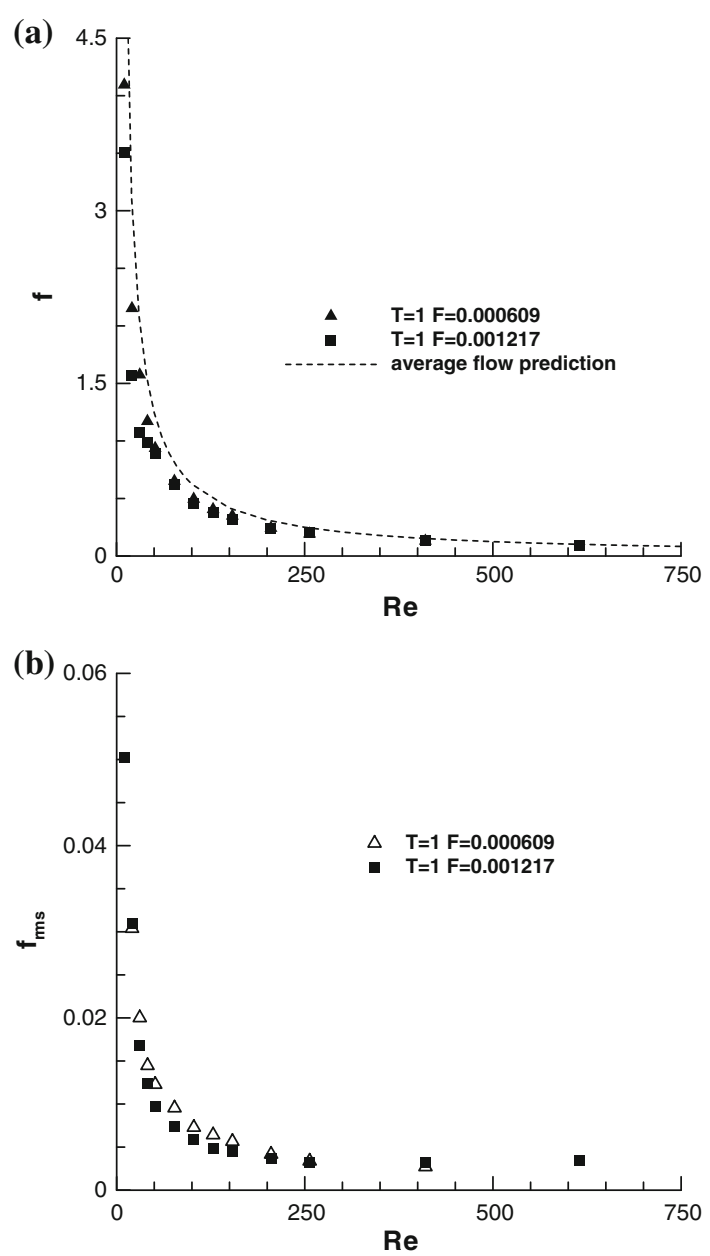

Fig. 15 Dependence of a average and b r.m.s. friction factor on average Reynolds number, with constant non-dimensional frequency and on-time to off-time ratio. The theoretical curve is also included as dashed line in a

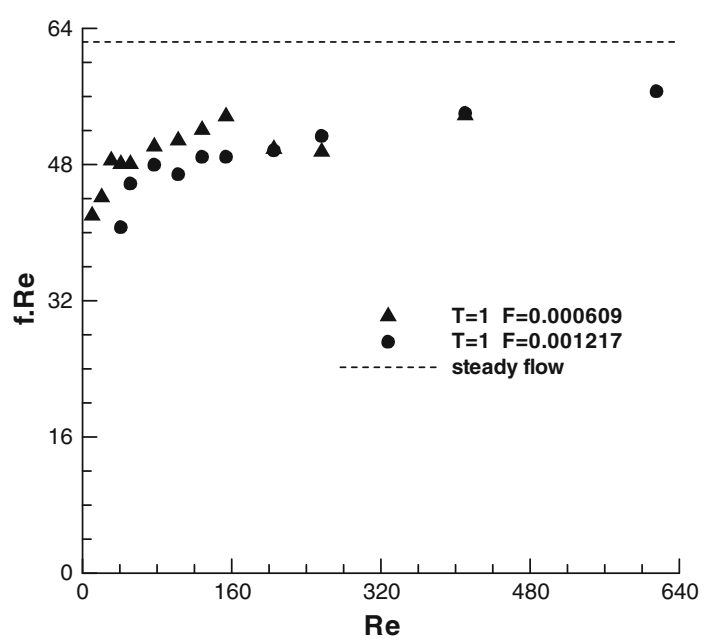

Fig. 16 Dependence of average friction factor times average Reynolds number on average Reynolds number, with constant nondimensional frequency and on-time to off-time ratio
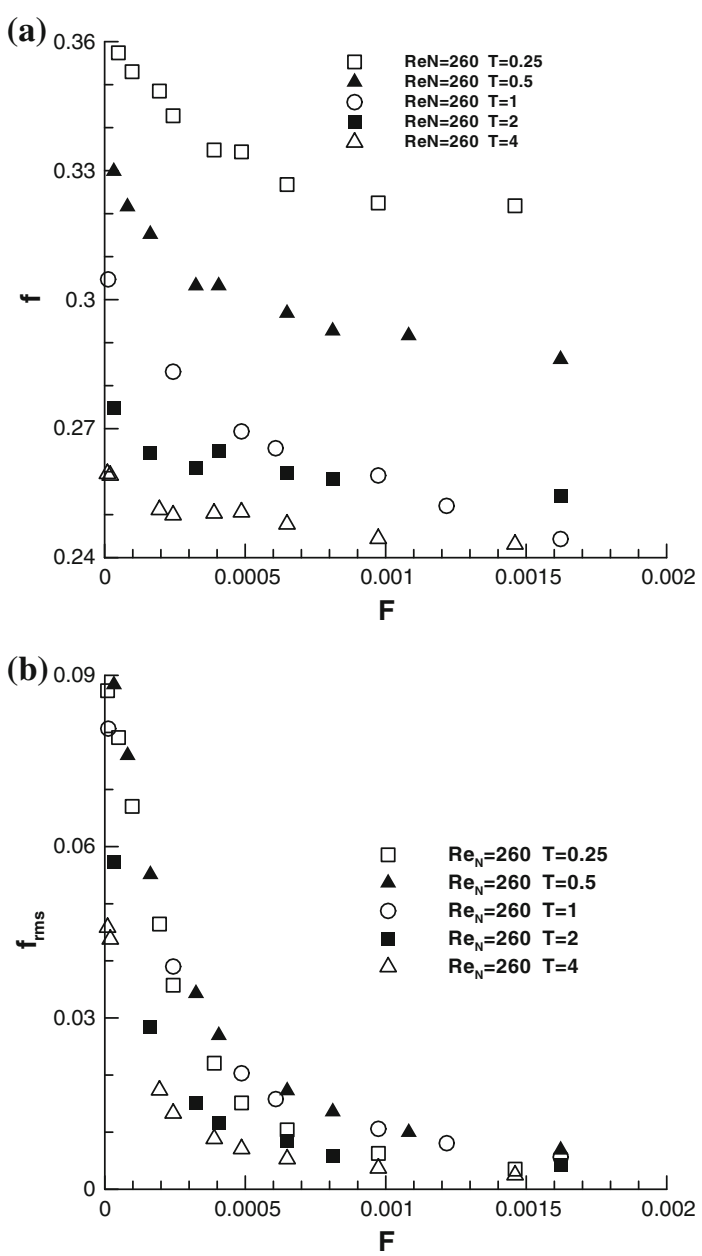

Fig. 17 Variation of a average and $\mathbf{b}$ r.m.s. friction factor with nondimensional frequency keeping the on-Reynolds number and ratio of on-time to off-time constant

increase in the average flow rate (or Reynolds number) with an increase in their ratio. There is also some saturation effect visible, whereby the friction factor becomes invariant of ontime, for larger on-time values. This is related to results obtained earlier in Figs. 10 and 13, where pressure increases monotonically with on-time at constant off-time and decreases monotonically and saturates with off-time at constant on-time. Changing the non-dimensional frequency, keeping the onReynolds number same reduces the friction factor and also makes the variation sharper. The friction factor r.m.s shows a non-monotonic variation. Going back to dimensional results in Figs. 11 and 14, we notice that the variation in pressure r.m.s is non-monotonic. Increasing the non-dimensional frequency reduces the value of friction factor r.m.s.

\section{A first-order model for pulsating flow}

The flow through each microchannel is modeled as a firstorder system, similar to a resistance-capacitance system in 

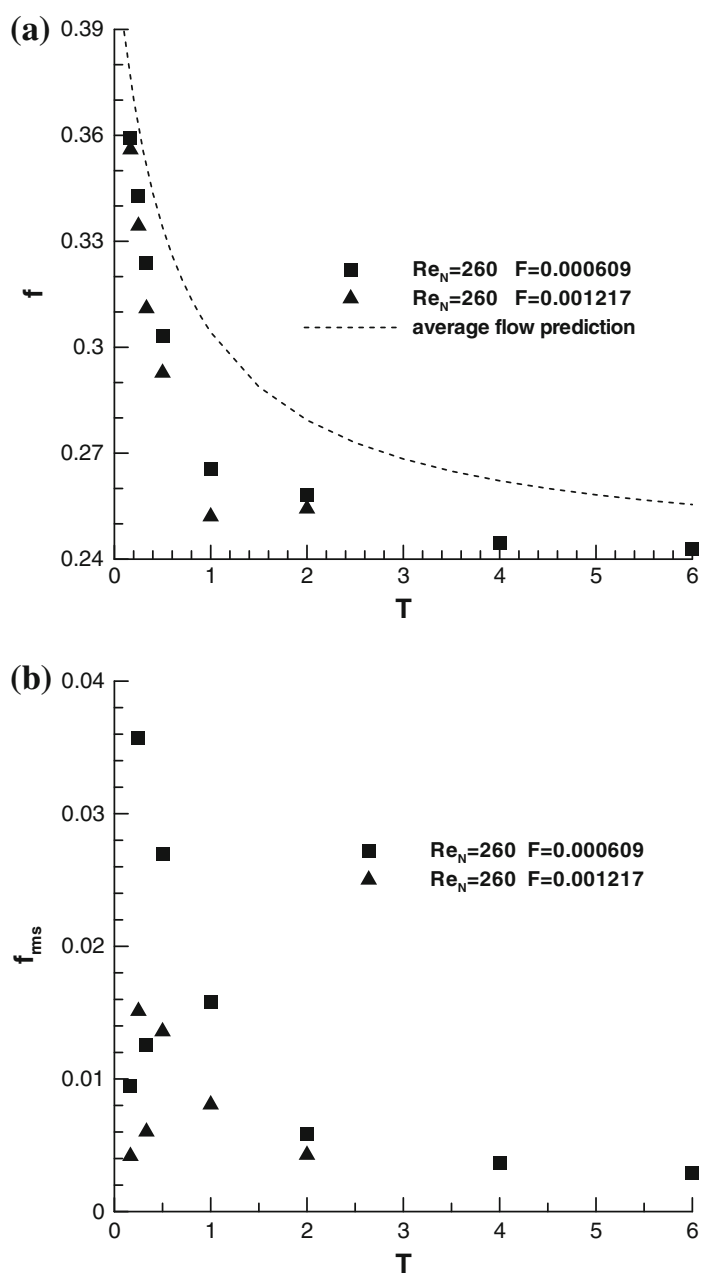

Fig. 18 Variation of $\mathbf{a}$ average and $\mathbf{b}$ r.m.s. friction factor with ratio of on-time to off-time keeping the on-Reynolds number and nondimensional frequency constant. The theoretical curve is also included as dashed line in $\mathbf{a}$

parallel as shown in Fig. 19a, with current acting as the equivalent of mass flow rate and voltage acting as equivalent of pressure.

The system equation, may, therefore, be written as

$i=\frac{V}{R}+C \frac{\mathrm{d} V}{\mathrm{~d} t}$.

With this modeling of the microchannels, the complete setup looks as shown in Fig. 19b, where the switch S is periodically closed and opened to generate current pulsations in the $R_{1}-C_{1}$ channel. The system may also be seen as two different systems acting alternately, each during the on (switch open) and off (switch closed) phases (Fig. 19c, d).

The system thus has two different time constants during the on- and off-phases. In the on-phase,

$\tau_{\mathrm{N}}=R_{1} C_{1}$

while, during the off-phase, the time constant is (see Fig. 19b)
$\tau_{\mathrm{F}}=R_{\mathrm{eq}} C_{\mathrm{eq}}=\frac{R_{1} R_{2}}{R_{1}+R_{2}}\left(C_{1}+C_{2}\right)$.

The first-order voltage drop across this system will be

ON phase: $V(t)=V_{\min }+\left(V_{\mathrm{ss}, \max }-V_{\min }\right)\left(1-e^{-\frac{t}{\tau_{\mathrm{N}}}}\right)$,

OFF phase: $V(t)=V_{\max }+\left(V_{\mathrm{ss}, \min }-V_{\max }\right)\left(1-e^{-\frac{t}{\tau_{\mathrm{F}}}}\right)$.

Note that the equations developed above are for a flowsplitter setup. The equations for a piston-based assembly are likely to be different and therefore the results between the two assemblies may differ. The proposed model is also different from that used by Morris and Forster (2004), because their system is pressure-driven whereas it is a mass-driven system in the present case. This point is further supported by $\mathrm{Ku}$ (1997). In practice, a resistance-capacitance-inductance system may be a still better model. In the present situation, however, the contribution of the effect of inductance on the system dynamics is negligible, because inductance will have effect only when for the mass flow rate changes, i.e., at the rising and falling edges of the pulses, which are of negligible duration here.

\subsection{Solution of equations}

The voltages may be normalized as

$v=\frac{V-V_{s s, \text { min }}}{V_{s s, \text { max }}-V_{s s, \text { min }}}$.

Therefore, the response in the normalized form will be,

ON phase: $v(t)=v_{\min }+\left(1-v_{\min }\right)\left(1-e^{-\frac{t}{\tau_{\mathrm{N}}}}\right)$,

OFF phase: $v(t)=v_{\max } e^{-\frac{t}{\tau_{\mathrm{F}}}}$.

Substituting the continuity condition, i.e., during the onphase, at $t=t_{\mathrm{N}}, v=v_{\max }$ and during the off-phase, at $t=t_{\mathrm{F}}, v=v_{\min }$, we have

$v_{\max }=v_{\min }+\left(1-v_{\min }\right)\left(1-e^{-\frac{t_{\mathrm{N}}}{\tau_{\mathrm{N}}}}\right)$

$v_{\min }=v_{\max } e^{-\frac{t_{\mathrm{F}}}{\tau_{\mathrm{F}}}}$

This gives,

$v_{\min }=\frac{e^{-\frac{t_{\mathrm{F}}}{\tau_{\mathrm{F}}}}\left(1-e^{-\frac{t_{\mathrm{N}}}{\tau_{\mathrm{N}}}}\right)}{1-e^{-\frac{t_{\mathrm{F}}}{\tau_{\mathrm{F}}}} e^{-\frac{t_{N}}{\tau_{\mathrm{N}}}}}$

$v_{\max }=\frac{1-e^{-\frac{t_{\mathrm{N}}}{\tau_{\mathrm{N}}}}}{1-e^{-\frac{t_{\mathrm{F}}}{\tau_{\mathrm{F}}}} e^{-\frac{t_{\mathrm{N}}}{\tau_{\mathrm{N}}}}}$.

Note that the rapid fall in pressure at the beginning of the off-cycle seen in experiments (see for example, Fig. 6) 
Fig. 19 Model of the system: a Single microchannel, b two microchannels in parallel, c circuit during on-phase, d equivalent circuit during off-phase (a)

(b)

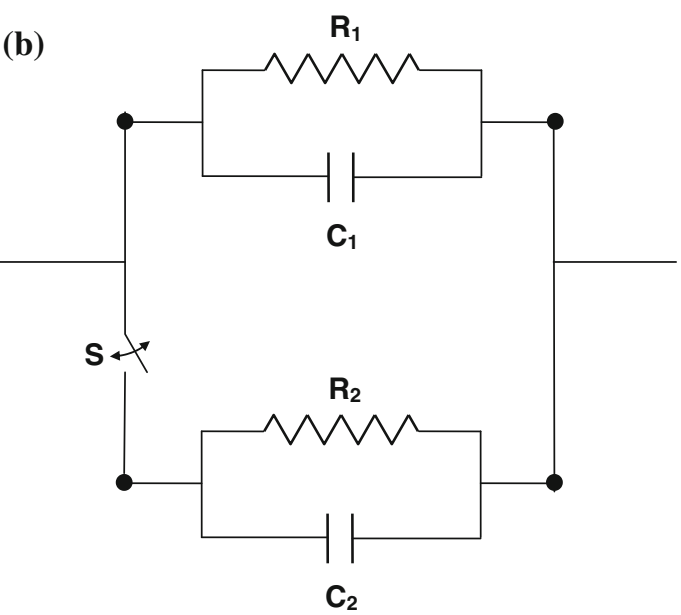

(c)

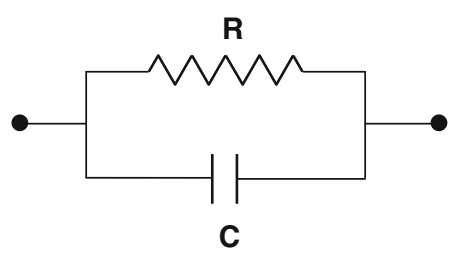

$\mathbf{R}_{1}$

i (t)

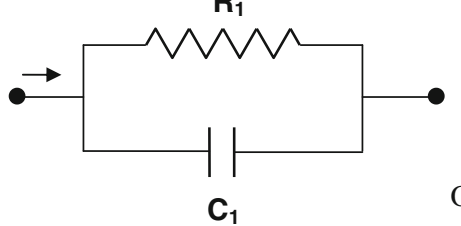

ON PHASE

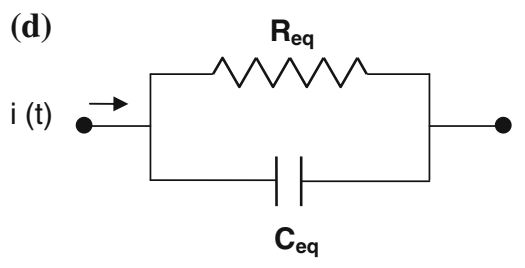

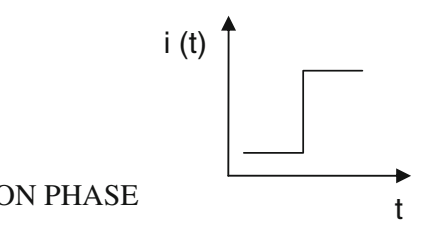

i (t)

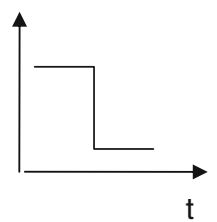

OFF PHASE

has not been modeled here; rather continuity between the two parts of the cycle has been assumed in deriving the above equations. This is because the magnitude of fall or the factors on which it depends are not clear. The model suggests that the capacitor $C_{1}$ can discharge suddenly upon connecting it to the other capacitor $C_{2}$. This discharge process is inadequately resolved in the measurements for most of the cases, and leads to appearance of two lines with different slopes during the off-phase.

The average voltage thus works out to be,

$$
\begin{aligned}
v_{\text {avg }} & =\frac{\int_{0}^{t_{\mathrm{N}}+t_{\mathrm{F}}} v(t) \mathrm{d} t}{t_{\mathrm{N}}+t_{\mathrm{F}}} \\
& =\frac{t_{\mathrm{N}}}{t_{\mathrm{N}}+t_{\mathrm{F}}}+\left(\tau_{\mathrm{F}}-\tau_{\mathrm{N}}\right) \frac{\left(1-e^{-\frac{t_{\mathrm{F}}}{\tau_{\mathrm{F}}}}\right)\left(1-e^{-\frac{t_{\mathrm{N}}}{\tau_{\mathrm{N}}}}\right)}{t_{\mathrm{N}}+t_{\mathrm{F}}} .
\end{aligned}
$$

It is interesting to note that the expression for average voltage is a sum of two terms: the first being the average value of the forced pulsations, while the second is a product of the system's first-order behavior. Notice that the second term will simply disappear if the time constants of the system in the on- and off-phases are equal. The second term also goes to zero for large values of on- and off-times, in which case the denominator becomes very large. In both these cases, the system will behave as though its average voltage is the same as the average voltage forced across it.

Similarly, the r.m.s. voltage turns out to be

$v_{\mathrm{rms}}=\sqrt{\frac{\int_{0}^{t_{\mathrm{N}}+t_{\mathrm{F}}}\left(v(t)-v_{\mathrm{avg}}\right)^{2} \mathrm{~d} t}{t_{\mathrm{N}}+t_{\mathrm{F}}}}=\sqrt{v_{\mathrm{rms}, \mathrm{ON}}^{2}+v_{\mathrm{rms}, \mathrm{OFF}}^{2}}$

where

$$
\begin{aligned}
v_{\mathrm{rms}, \mathrm{ON}}^{2}= & \frac{\tau_{\mathrm{N}}}{t_{\mathrm{N}}+t_{\mathrm{F}}}\left[\left(1-v_{\mathrm{avg}}\right)^{2} \frac{t_{\mathrm{N}}}{\tau_{\mathrm{N}}}+\frac{1}{2}\left(1-v_{\mathrm{min}}\right)^{2}\right. \\
& \left.\times\left(1-e^{-2 \frac{t_{\mathrm{N}}}{\tau_{\mathrm{N}}}}\right)-2\left(1-v_{\mathrm{avg}}\right)\left(1-v_{\mathrm{min}}\right)\left(1-e^{-\frac{t_{\mathrm{N}}}{\tau_{\mathrm{N}}}}\right)\right]
\end{aligned}
$$

$$
\begin{aligned}
v_{\mathrm{rms}, \mathrm{OFF}}^{2}=\frac{\tau_{\mathrm{F}}}{t_{\mathrm{N}}+t_{\mathrm{F}}} & {\left[v_{\mathrm{avg}}^{2} \frac{t_{\mathrm{F}}}{\tau_{\mathrm{F}}}+\frac{1}{2} v_{\max }^{2}\left(1-e^{-2 \frac{t_{\mathrm{F}}}{\tau_{\mathrm{F}}}}\right)\right.} \\
& \left.-2 v_{\mathrm{avg}} v_{\max }\left(1-e^{-\frac{t_{\mathrm{F}}}{\tau_{\mathrm{F}}}}\right)\right]
\end{aligned}
$$




\subsection{Model results}

The model parameters for the present experimental conditions were first estimated. The product of resistance and capacitance is equal to the response time (see Eqs. 10 and 11) and can be estimated by running experiments for long duration of on- and off-times; however, we have not tried to determine the individual values of resistance and capacitance. Notice that only $\tau_{\mathrm{N}}$ and $\tau_{\mathrm{F}}$ enter the equations and therefore these values are adequate for the calculations. The obtained values are $\tau_{\mathrm{N}}=72 \mathrm{~s}$ and $\tau_{\mathrm{F}}=48 \mathrm{~s}$.

The model result for a square waveform as a function of flow rate is shown in Fig. 4. The model result compares well with the experimental data. Note that the model results are closer to the experimental data as compared to the theoretical curve, highlighting the importance of modeling in the present case.

The model results for a constant $t_{\mathrm{F}}$ and $t_{\mathrm{N}}$ varying are shown in Figs. 10 and 11. Again the model result is closer to the experimental data then the theoretical curve (Fig. 10). It is further reassuring that a qualitatively correct behavior is seen for r.m.s. (Fig. 11). The model is able to capture the existence and location of peak in r.m.s. correctly in Fig. 11b. However, it fails to capture the existence of the peak in Fig. 11a; this may be due to the fact that the model ignores the sudden pressure drop at the start of the off-phase, as discussed in Sect. 5.1. The model shows that the pressure r.m.s. asymptotes to a constant value. An asymptotically constant value is, however, not experimentally seen, due to the limited range of $t_{\mathrm{N}}$ value employed in the experiment. As noted in Sect. 3.3, both the minimum and maximum pressure values increase monotonically with an increase in the on-time, but the maximum value increases more than the minimum value; this feature is also reproduced well by the model (not shown).

The model predicts results for a constant $t_{\mathrm{N}}$ and $t_{\mathrm{F}}$ varying case reasonably well (Figs. 13, 14). The model, however, shows only a single peak in pressure r.m.s., as opposed to two peaks in the experiments. The results with total time period varying for square pulsations from the model are shown in Figs. 7 and 8. Here, the variation in r.m.s. is found to agree better than mean when compared against the experiments.

The results in this section suggest that the overall agreement between model and experiment is satisfactory. The comparison can be improved by further modeling the rapid discharge of capacitor $C_{1}$ at the beginning of the off-phase.

\section{Discussion}

The results in this paper suggest that the pressure drop in microchannel lies between the two steady state values, corresponding to the maximum and minimum mass flow rates through the microchannel. The pressure is, however, observed to lie closer to the lower steady state value, for almost all cases investigated herein.

The average pressure drop reduces upon applying pulsations to the flow. This reduction is brought about because pressure takes time to build-up upon increasing the flow rate, whereas the pressure drops rapidly upon reducing the mass flow rate. The deviation in pressure drop with pulsations as compared to steady state value increases with an increase in frequency and a reduction in flow rate. In other words, this may be due to the shape of the pressure waveform. This suggests that inertia is important during the acceleration phase (rising part of the rectangular pulse), but not particularly so during the deceleration phase. The average velocity reduces during the falling part of the rectangular pulse, and is expected to lead to a reduction in velocity throughout the cross-section of the channel. This may lead to a reversal in velocity near the wall, possibly leading to a change in direction of the wall shear stress and an undershoot in pressure (Das and Arakeri 2000). Experimental evidence of a reversal in velocity profile for a mass-driven system is provided by Muta and Nakane (1980). The theoretical work of Ohmi and Iguchi (1981) suggests that the instantaneous friction factor is almost the same as steady friction factor for quasi-steady regime. The instantaneous friction factor is always larger than the steady value during the acceleration phase of the intermediate regime; it can, however, be larger, equal or smaller during the deceleration phase of the intermediate regime and in the inertial dominant regime. A second reason for this interesting behavior is provided by the model, in that the capacitor can discharge rapidly at the beginning of offphase. This leads to a sudden reduction in pressure and contributes to a smaller average pressure value over the cycle.

The importance of inertial force as compared to other forces may change with the channel size. It is, however, not clear if the reduction in pressure drop with pulsation frequency seen here is a scale effect; lack of corresponding data in larger size channels precludes a comparison. It would therefore be worthwhile to study this phenomenon as a function of channel size and geometry (cross-section). This may also help answer if large pressure drop expected during blood flow in fine veins and arteries, in human and animal bodies, is partly offset by the pulsating effect of the flow. The pressure drop penalty is particularly crucial for smaller veins and arteries due to their small diameter and relatively large numbers (Nichols and O'Rourke 1990; Ku 1997).

It is normally argued (see example, Uchida 1956) that pressure drop should increase with pulsations, because additional pressure is required to overcome the inertial 
forces in the system. Whereas this argument applies to pressure-driven systems, it does not appear to hold for mass-driven systems. In the latter case, as already mentioned, there is a slow increase in pressure during the acceleration phase which coupled with a rapid decrease during the deceleration phase, leads to a bias of the pressure drop towards a smaller average. This asymmetry is crucial for a difference in behavior with respect to their steady counterparts, and underscores the importance of obtaining temporally resolved data for a proper understanding of the flow characteristics. The model presented here also helps to bring out the difference between pressure-driven and mass-driven systems.

Based on data in this paper, we propose the following correlation of friction factor in terms of the other three nondimensional parameters:

$f=28.17 R e^{-0.91} T^{-0.0004} F^{-0.031}$

The above correlation fits most of the experimental data within 5\% error (Fig. 20). Note that Eq. 19 is strictly valid only in the range for which experimental information is available (i.e., $R e=10-620, F=0-0.00365, T=0.17-6$ ) and should be applied with care for parameter values outside this range. Note the small exponent of $T$ which suggests that $f$ is practically independent of the duty cycle. From the above correlation, one sees that $f \cdot R e$ varies as $D_{\mathrm{h}}^{0.028}$, which suggests a reduction in pressure drop with a decrease in hydraulic diameter, keeping the frequency, average velocity and fluid, the same. This would suggest a scale effect on the flow with pulsations. Note, however, that $D_{\mathrm{h}}$ has not been varied in this study, and therefore this observation has to be taken with a pinch of salt. It would nonetheless be useful to check this finding because of its potential practical applications.

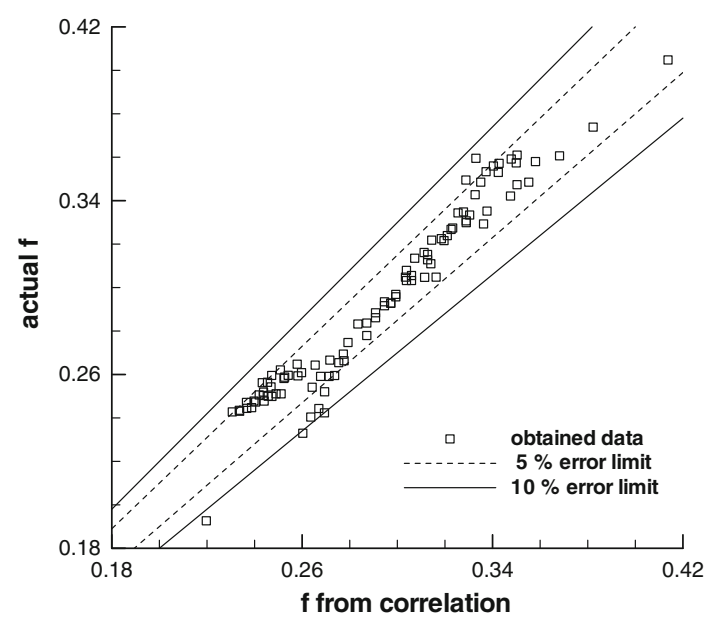

Fig. 20 Comparison between the values of friction factor obtained by measurement against that predicted by correlation. The total number of data points shown here is 95

\section{Conclusions}

Pulsatile flow in microchannels is of interest because of its relevance to flow in microdevices, besides being of relevance for several engineering problems, and is studied in detail in this work. The average and r.m.s. pressure drop as a function of mass flow rate, pulsation frequency, and duty cycle have been investigated here; both dimensional and non-dimensional results have been presented. Although a direct comparison of these results is not possible because of paucity of corresponding results at microscales in the literature, discussion and comparison is provided as appropriate. Further, theoretical and modeling results are offered and good agreement of the experimental data with these improves our confidence in the data and analysis.

The average pressure drop increases linearly with flow rate, while the pressure r.m.s. increases quadratically. The average pressure drop decreases with an increase in frequency; the maximum reduction being about $33 \%$ with respect to the steady state value. On the other hand, the pressure r.m.s. increases linearly with period of pulsation and varies non-monotonically with duty cycle.

It is also shown that the flow can be modeled as a firstorder system. Both the mean and r.m.s. pressure drop predicted from the model compare reasonably well against the experimental data. The results are significant because they bring about some important differences between pressure-driven and mass-driven systems, such as lower pressure drop with pulsations as compared to their steady counterparts. The proposed correlation would help to quantify pressure drop in rectangular microchannels, at least for the parameter range covered in this study. This detailed study therefore supplements other application oriented studies involving such time-varying flows in microdevices.

\section{References}

Abdullah M, Duwairi HM (2008a) Thermal and flow analysis of twodimensional fully developed flow in an AC magneto-hydrodynamic micropump. Microsyst Technol 14(8):1117-1123

Abdullah M, Duwairi HM (2008b) Numerical computation of twodimensional flow in an AC magneto hydrodynamic micropump. Can J Phys 86(11):1321-1325

Brereton GJ (2009) Approximate behavior of arbitrarily unsteady laminar flow in long, straight, flexible tubes. Phys Fluids 21:081902

Çarpinlioglu MO, Gündogdu MY (2001) A critical review on pulsatile pipe flow studies directing towards future research topics. Flow Meas Instrum 12:163-174

Chakraborty S, Bhalla APS (2007) Controlling microchannel gas flow rates through time-modulated pressure pulsation. J Appl Phys 102:114910 
Chakraborty S, Das T, Chattoraj S (2007) A generalized model for probing frictional characteristics of pressure-driven liquid microflows. J Appl Phys 102:104907

Das D, Arakeri JH (2000) Unsteady laminar duct flow with a given volume flow rate variation. J Appl Mech 67:274-281

Duwairi HM, Abdullah M (2007) Thermal and flow analysis of a magneto-hydrodynamic micropump. Microsyst Technol 13(1):33-39

Duwairi HM, Abdullah M (2008) Numerical computation of fluid flow in a magnetohydrodynamic micropump. Turkish J Eng Env Sci 32:1-5

Glasgow I, Aubry N (2003) Enhancement of microfluidic mixing using time pulsing. Lab Chip 3:114-120

Glasgow I, Lieber S, Aubry N (2004a) Parameters influencing pulsed flow mixing in microchannels. Anal Chem 76:4825-4832

Glasgow I, Batton J, Aubry N (2004b) Electroosmotic mixing in microchannels. Lab Chip 4:558-562

Gu W, Zhu X, Futal N, Cho BS, Takayama S (2004) Computerized microfluidic cell culture using elastomeric channels and Braille displays. PNAS 101:15861-15866

Haddad K, Ertunc O, Mishra M, Delgado A (2010) Pulsating laminar fully developed channel and pipe flows. Phys Rev E 81:013603

Havemann HA, Rao NNN (1954) Heat transfer in pulsating flow. Nature 174:41

He X, Ku DN (1994) Unsteady entrance flow development in a straight tube. J Biomech Eng 116(3):355-361

Ku DN (1997) Blood flow in arteries. Annu Rev Fluid Mech 29:399434

Lee S-Y, Wereley ST, Gui L, Qu W, Mudawar I (2002) Microchannel flow measurement using particle image velocimetry. In: Proceedings of IMECE, New Orleans, Louisiana, USA, 17-22 November

Lee S-Y, Jang J, Wereley ST (2008) Effects of planar inlet plenums on the hydrodynamically developing flows in rectangular microchannels of complementary aspect ratios. Microfluid Nanofluid 5:1-12

Morini GL (2004) Laminar liquid flow through silicon microchannels. J Fluid Eng 126:485-489

Morris CJ, Forster FK (2004) Oscillatory flow in microchannels: comparison of exact and approximate impedance models with experiments. Exp Fluids 36:928-937

Muta T, Nakane K (1980) Unsteady flow in circular tube (velocity distribution in pulsating flow). Bull JSME 123:1990-1996
Nichols WW, O'Rourke MF (1990) McDonald's blood flow in arteries, 3rd Ed edn. Edward Arnold, London

Ohmi M, Iguchi M (1981) Flow pattern and frictional losses in pulsatile pipe flow. Part 6: frictional losses in a laminar flow. Bull JSME 24:1756-1763

Ohmi M, Iguchi M, Usui T (1981) Flow pattern and frictional losses in pulsatile pipe flow. Part 5: wall shear stress and wall pattern in a laminar flow. Bull JSME 24:75-81

Singh SG, Kulkarni A, Duttagupta SP, Puranik BP, Agrawal A (2008) Impact of aspect ratio on flow boiling of water in rectangular microchannels. Exp Thermal Fluid Sci 33:153-160

Singh SG, Bhide R, Duttagupta SP, Puranik BP, Agrawal A (2009) Two-phase flow pressure drop characteristics in trapezoidal silicon microchannels. IEEE Trans Compon Packag Technol 32:887-900

Steinke ME, Kandlikar SG (2004) Single phase heat transfer enhancement techniques in microchannel and minichannel flows. In: International conference on microchannels and minichannels, Rochester, New York, USA, 17-19 June

Stroock AD, Dertinger SK W, Ajdari A, Meziç I, Stone HA, Whitesides GM (2002) Chaotic mixer for microchannels. Science 295:647-651

Tang GH, Li Z, Wang JK, He YL, Tao WQ (2006) Electroosmotic flow and mixing in microchannels with lattice Boltzmann method. J Appl Phys 100:1-10

Uchida S (1956) The pulsating viscous flow superposed on the steady laminar motion of incompressible fluid in a circular pipe. ZAMP $7: 403-422$

Unger MA, Chou HP, Thorsen T, Scherer A, Quake SR (2000) Monolithic microfabricated valves and pumps by multilayer soft lithography. Science 288:113-116

Ünsal B, Ray S, Durst F, Ertunç Ö (2005) Pulsating laminar pipe flows with sinusoidal mass flux variations. Fluid Dyn Res 37(2005):317-333

Wu YH, Wiwatanapataphee B, Hu M (2008) Pressure-driven transient flows of Newtonian fluids through microtubes with slip boundary. Phys A 387:5979-5990

Yakhot A, Arad M, Ben-Dor G (1999) Numerical investigation of a laminar pulsating flow in a rectangular duct. Int $\mathrm{J}$ Numer Methods Fluids 29:935-950 